\title{
A NEAR INFRARED SURVEY OF THE LMC
}

\author{
TAKEHIKO WADA AND MUNETAKA UENO \\ Dept. of Earth Science \& Astronomy, Univ. of Tokyo,Japan \\ AND \\ TOSHIKAZU EBISUZAKI AND YOSUKE OHNO \\ Computational Science Lab., The Institute of Physical \\ and Chemical Research, Japan
}

We surveyed the central $3^{\circ} \times 6^{\circ}$ region of the Large Magellanic Cloud (LMC) with angular resolution of $10^{\prime \prime} .0$ at the $\mathrm{J}(1.25 \mu \mathrm{m}), \mathrm{H}(1.65 \mu \mathrm{m})$ and $\mathrm{K}^{\prime}(2.15 \mu \mathrm{m})$ bands. The observations were performed from Oct to Nov, 1994 at the Siding Spring Observatory. We used a $25 \mathrm{~cm} / F 3.5$ Newtonian telescope equipped with a $512 \times 512 \mathrm{PtSi}-\mathrm{Camera}$ (Ueno et al. 1992). The plate scale was $4 . " 6 \times 6 . " 0 /$ pixel and the total field of view was $40 .^{\prime} 2 \times 52 .^{\prime} 3$. The system was attached onto the Automated Patrol Telescope of the University of New South Wales. The limiting magnitudes of the survey were 13.6, 11.9, and 10.0 magnitude $(3 \sigma)$ at the $\mathrm{J}, \mathrm{H}$, and $\mathrm{K}^{\prime}$ bands, respectively. The positions and the $\mathrm{J}, \mathrm{H}$, and $\mathrm{K}^{\prime}$ magnitudes were derived for 1599 point sources whose $\mathrm{S} / \mathrm{N}$ ratio were more than four at the $\mathrm{K}^{\prime}$-band. The dominant components of the sources are red super giants (RSG), luminous M-type giants and AGB stars in the LMC. The distributions of RSG, luminous giants and $\mathrm{CO}$ line flux are spatially different from each other. This supports the idea that the position of the active star forming region has changed during the past $10^{8}$ years. The detected sources are cross-identified with the IRAS Point Source Catalog. Sixty-two of 680 IRAS sources in the region spatially coincide with the detected sources and the spectrum energy distributions from $1.25 \mu \mathrm{m}$ to $100 \mu \mathrm{m}$ were derived for these 62 sources. Half of them are well described by a black body spectrum, while the others show a flat or redder spectrum. These latter sources are supposed to be stars with dust envelopes.

\section{References}

Ueno, M., Ito, M., Kasaba, Y., \& Sato, S. 1992, SPIE, 1762, 423 